\title{
2 Ziele und Fragestellungen
}

Aus der Literatur hinsichtlich der Transitionsthematik geht hervor, dass der eigenverantwortliche Umgang mit der Erkrankung eine große Herausforderung für die Betroffenen darstellt und als eine wichtige Voraussetzung für ein erfolgreiches Transitieren von der Pädiatrie in die Erwachsenversorgung gesehen wird (Oldhafer, 2016). Des Weiteren wird beschrieben, dass zum Zeitpunkt der Volljährigkeit häufig keine ausreichenden, auf die Transition bezogenen Kompetenzen vorliegen. Dies wird sowohl durch krankheitsbedingte Entwicklungsverzögerungen, als auch durch Überbehütung von Seiten der Eltern sowie Pädiatern begründet (ebd.). Daneben findet die subjektive Bedürfnislage der Betroffenen selbst in der Literatur kaum Berücksichtigung (u.a. Gelbmann \& Melter, 2010; Keller, 2010; Kreuzer et al., 2014; Kreuzer et al., 2015; Minden \& Niewerth, 2015; Radke, 2015; Reisch \& Reincke, 2014; Sachverständigenrat zur Begutachtung der Entwicklung im Gesundheitswesen, 2009).

Folglich ist das Ziel der vorliegenden Arbeit, fehlende notwendige Kompetenzen der Betroffenen in Deutschland zu identifizieren sowie deren Wünsche hinsichtlich der eigenen Transition zu erheben, um eine individuelle, alters- und entwicklungsgerechte Betreuung für die Jugendlichen und jungen Erwachsenen mit chronischer Erkrankung sicher zu stellen. In diesem Zusammenhang sollen auch entwicklungspsychologische Fragestellungen sowie genderspezifische Aspekte Beachtung finden. Des Weiteren soll auf Grundlage der Ergebnisse herausgefiltert werden, wie Programme oder Schulungen als Unterstützungsmaßnahme aus Sicht der Betroffenen gestaltet und wie Gesundheitskompetenzen in diesem Rahmen vermittelt werden können. Konkret ergeben sich daraus folgende Fragestellungen:

- Welche gesundheitsrelevanten Transitionskompetenzen haben Jugendliche und junge Erwachsene mit chronischer Erkrankung?

- Welche Bedürfnisse und Wünsche stellen Jugendliche und junge Erwachsene mit chronischer Erkrankung an die Transition? 